\title{
Low risk patients with acute atrial fibrillation and elevated high-sensitivity troponin do not have increased incidence of pathological stress test: a prospective cohort study
}

Johan Thelin ( $\square$ johan.thelin@skane.se )

Department of Clinical Sciences, Lund University, Sweden https://orcid.org/0000-0002-6101-4946

Sofia Gerward

Department of Clinical Sciences, Lund University

Olle Melander

Department of Clinical Sciences

Research article

Keywords: atrial fibrillation; troponin; myocardial injury; coronary artery disease; stress test

Posted Date: October 14th, 2019

DOI: https://doi.org/10.21203/rs.2.11487/v2

License: (c) (i) This work is licensed under a Creative Commons Attribution 4.0 International License.

Read Full License 


\section{Abstract}

Background Many patients with atrial fibrillation (AF) or atrial flutter (AFL) and rapid ventricular response (RVR) have elevated high-sensitivity troponin T (hsTnT) values. Elevated hsTnT is an independent risk marker for cardiovascular events and mortality. The aim was to examine if AF/AFL patients with RVR and elevated hsTnT have an increased incidence of pathological cardiac stress tests, indicating need of further evaluation for coronary artery disease (CAD). Methods We prospectively included 90 AF/AFL patients without known heart failure and CAD presenting with AF/AFL and RVR. Half of the patients had elevated hsTnT (cases) and half had levels below the 99th percentile (controls). All patients were discharged in sinus rhythm. After approximately one week in sinus rhythm a new hsTnT was analysed and the patients performed a bicycle exercise stress test within the 30 day follow-up. The primary endpoint was a pathological stress test confirmed by a pathological SPECT myocardial perfusion imaging or a coronary angiography. Results None of the controls reached the primary endpoint. Two patients ( $4 \%$ ) out of the 45 cases reached the primary endpoint ( $p=0.49$ vs controls), but only one was found to have significant $C A D$ at subsequent coronary angiography. Conclusions Patients with paroxysmal AF/AFL, without history of CAD and heart failure, who present with a RVR and minor hsTnT elevations do not have an increased incidence of pathological stress test compared to patients with hsTnT values below the 99th percentile.

\section{Background}

Atrial fibrillation (AF) and atrial flutter (AFL) are the most common arrhythmias in the emergency department (ED) [1]. Many of these patients present with a rapid heart rate and have symptoms like palpitations, chest pain, dyspnea, dizziness or fatigue [2]. As a consequence cardiac troponins are often analysed in AF/AFL patients with rapid ventricular response (RVR) [3].

High-sensitivity troponin (hsTn) assays have improved the early diagnosis of acute coronary syndrome (ACS) $[4,5]$ and has also proven to be a strong independent risk factor for cardiovascular events and mortality [6]. The more sensitive assays have also increased the number of AF/AFL patients with minor troponin elevations above the 99th percentile. The causes and significance of these elevations and how to best handle these patients in clinical practise are largely unknown [7].

Some studies suggest that patients with AF have an increased prevalence of subclinical coronary artery disease (CAD) [8, 9] and an increased risk of myocardial infarction (MI) [10]. However, it is not yet known if elevated troponin in AF/AFL patients with RVR might be due to a significant CAD causing supplydemand mismatch and relative myocardial ischemia in the setting of a rapid heart rate.

The primary aim of this study was to examine if AF/AFL patients without known heart failure and CAD who present with RVR and elevated high-sensitivity troponin T (hsTnT) have an increased incidence of pathological cardiac stress test compared to patients with hsTnT values below the 99th percentile. If the 
hypothesis proves correct, troponin elevations in this context may indicate need of further evaluation for CAD.

Our secondary aims were to analyse which background factors and clinical parameters differ between patients with elevated hsTnT and patients with hsTnT values below the 99th percentile and to register the occurrence of any adverse events during follow-up.

\section{Methods}

\section{Study design and setting}

This prospective study was conducted at the Skåne University Hospital in Lund and Malmö, Sweden. We included patients $\geq 40$ years old presenting in the ED with a primary diagnosis of AF or AFL and a heart rate $\geq 110$ beats/min and who had at least one hsTnT sample analysed. The patients had to be discharged in sinus rhythm and be able to perform a bicycle exercise stress test within 30 days.

We excluded patients with other possible explanations for hsTnT elevation than tachycardia related to AF/AFL. Patients with history of CAD, heart failure (HF), significant anaemia, hypotension (systolic BP < $90 \mathrm{mmHg}$ ), hypoxia (saO2 <90\%), renal failure (creatinine $>200 \mu \mathrm{g} / \mathrm{L}$ ), acute infection, rhabdomyolysis (myoglobin $>250 \mu \mathrm{g} / \mathrm{L}$ ), thyrotoxicosis, acute ischemic stroke or intracranial haemorrhage or acute pulmonary embolism, were excluded. History of CAD was defined as prior percutaneous coronary intervention $(\mathrm{PCl})$, coronary artery bypass grafting (CABG), a > $50 \%$ stenosis on coronary angiography or having had a myocardial infarction. Known HF was defined as documented ejection fraction $<45 \%$, moderate to severe valvular heart disease, hypertrophic cardiomyopathy or dilated cardiomyopathy. Significant anaemia was defined as haemoglobin values $<90 \mathrm{~g} / \mathrm{L}$ for males and $<80 \mathrm{~g} / \mathrm{L}$ for females at inclusion. Acute infection was defined as CRP $>50 \mathrm{mg} / \mathrm{L}$ and/or temperature $>38^{\circ} \mathrm{C}$. Patients with left bundle branch block (LBBB) on ECG were also excluded because of the difficulty to interpret ST-T changes during stress testing in these patients.

In line with the power calculation (see below), we included 45 patients with elevated hsTnT (cases) and 45 patients with hsTnT values below the 99th percentile (controls). Patients eligible for inclusion were identified and enrolled after ED triage when the corresponding author or research nurses were available (convenience sample), during the time period 14th April, 2015 to 22nd October, 2018.

Data including echocardiography examinations, ECG, laboratory results, clinical and background variables was extracted from medical records according to a predefined protocol and the patients also completed a questionnaire at inclusion.

To differ between acute and chronic hsTnT elevations we analysed a second hsTnT sample after about a week in sinus rhythm. Patients were scheduled for an outpatient bicycle exercise stress test within 30 days after inclusion. If a single-photon emission computed tomography (SPECT) myocardial perfusion 
imaging already was scheduled or performed before the planed bicycle exercise test, the bicycle exercise test was considered unnecessary and cancelled. Patients with an inconclusive bicycle exercise test were referred to a subsequent standard SPECT myocardial perfusion imaging study [11].

The follow-up period for incidence of a first major adverse cardiac event (MACE) or death was 30 days from inclusion and data on these events were retrieved from medical records.

All patients gave their informed consent. The study was conducted according to the principles of the Declaration of Helsinki and approved by the Regional Ethics Committee in Lund, Lund University (registry number 2014/453).

\section{Primary endpoint}

The primary endpoint was a pathological stress test confirmed by a pathological SPECT myocardial perfusion imaging or a coronary angiography depending on clinical indication.

\section{Definitions and troponin $T$ analysis}

Hypertension and hyperlipidemia were defined as ongoing medication for each condition. Data on diabetes, current smoking, prior AF or AFL and prior stroke or transient ischemic attack (TIA) were based on both patient questionnaires and medical records. Chest pain was defined as any chest discomfort described as more than mild. Significant ST depression and significant hsTnT dynamic change were defined according to current guidelines [12-14]. During tachycardia a new horizontal or down-sloping ST depression $\geq 1 \mathrm{~mm}$ in any lead was considered significant and in patients with elevated hsTnT at inclusion a $>20 \%$ change compared to follow-up hsTnT analysis after a about a week was considered significant. The secondary endpoint of MACE during 30 days of follow-up was defined as a first ACS, PCI, CABG or death.

The hsTnT method used was Roche (Roche Diagnostics, Basel, Switzerland) high-sensitivity troponin T assay, with a detection limit of $5 \mathrm{ng} / \mathrm{L}$ and a 99th percentile cut-off point of $14 \mathrm{ng} / \mathrm{L}$.

Bicycle exercise stress test

The bicycle exercise test was performed on a Monark 939E ergometer bicycle following the protocol used in standard care with gradually increasing work load and subsequent rest $[15,16]$. During the entire exercise protocol patients were monitored with a continuous 12-lead ECG, repeated systolic blood pressure measurements and repeated registrations of symptoms (chest pain and dyspnea) according to validated rating scales [17]. The test was considered pathological if there was a planar or down-sloping ST segment depression of $>1 \mathrm{~mm}, 60 \mathrm{~mm}$ after the $\mathrm{j}$ point in any lead or if the test had to be interrupted due to severe chest pain, systolic blood pressure drop $\geq 15 \mathrm{mmHg}$, severe ventricular arrhythmia or significant ST elevations. The test was considered inconclusive if the patients were unable to reach $\geq$ $85 \%$ of age-predicted maximum heart rate, if the patient had significant ventricular arrhythmia (i.e. $\geq 3$ consecutive ventricular ectopic beats (VEBs) or increased frequency of VEBs during exercise) or if the ST segment deviation and/or patients symptoms were judged difficult to interpret $[13,18]$. The physicians 
who performed the bicycle stress tests were not informed about the patients' hsTnT values in the referral texts.

\section{Statistical analysis}

A power calculation with an alpha risk of 0.05 and a power of 0.80 was performed. In studies including individuals without suspicion of CAD the prevalence of unknown significant CAD has been reported to be $7 \%$ [19] and the incidence of pathological stress test has been reported to be $8 \%$ [20]. Based on this we estimated the incidence of pathological stress test to approximately $7 \%$ in the control group and we assumed a clinically highly relevant incidence of $30 \%$ in the case group. This resulted in a required sample size of at least 45 patients in each group.

Continuous variables are presented as medians with the interquartile range (IQR) and compared with the Mann- Whitney test. Categorical variables are presented as numbers and percentages and compared using the chi-square test or Fischer's exact test if the expected count was low $<5$.

Multivariable logistic regression analyses with elevated hsTnT at inclusion as dependent variable and clinically relevant background factors as covariates were performed. The results are presented as odds ratios (OR) with $95 \%$ confidence intervals $(\mathrm{Cl})$.

All tests were two tailed. Data management and statistical analysis were performed using IBM SPSS Statistics, version 22.

\section{Results}

Of 124 patients eligible for inclusion 18 declined (10 cases and 8 controls) to participate and 16 dropped out during the study period ( 7 cases and 9 controls), which resulted in a total of 90 patients ( 45 controls and 45 cases) who completed the study protocol. The baseline characteristics and clinical variables at presentation are shown in Table 1. In summary patients with elevated hsTnT were older and had more comorbidities, resulting in a higher CHA2DS2-VASc score. Cardioversion was performed in 35 controls (electrical cardioversion $89 \%$ and pharmacological cardioversion with vernakalant $11 \%$ ) and in 32 cases (electrical cardioversion $75 \%$, pharmacological cardioversion with vernakalant $22 \%$ and pharmacological cardioversion with flecainide $3 \%$ ). Echocardiography data were available in $86 \%$ of the patients and no one had reduced ejection fraction.

Table 1.

Baseline characteristics and clinical variables at presentation 


\begin{tabular}{lccc}
\hline & Controls $(\mathbf{n}=\mathbf{4 5})$ & Elevated hsTnT (n=45) & p-value \\
\hline Age (years) & $62(56-70)$ & $70(66-76)$ & $<0.001$ \\
Male sex & $24(53 \%)$ & $23(51 \%)$ & 0.83 \\
\hline Current smoking & $4(9 \%)$ & $4(9 \%)$ & 1.0 \\
\hline Hypertension & $21(47 \%)$ & $26(58 \%)$ & 0.29 \\
\hline Hyperlipidemia & $6(13 \%)$ & $16(36 \%)$ & 0.014 \\
\hline Diabetes & $0(0 \%)$ & $10(22 \%)$ & $\mathbf{0 . 0 0 1}$ \\
\hline Prior stroke/TIA & $1(2 \%)$ & $7(16 \%)$ & 0.06 \\
\hline Prior AF/AFL & $32(71 \%)$ & $30(67 \%)$ & 0.65 \\
\hline $\mathrm{CHA}_{2} \mathrm{DS}_{2}$ VASc (median, range) & $1(0-4)$ & $3(0-7)$ & $<0.001$ \\
\hline Atrial fibrillation & $40(89 \%)$ & $36(80 \%)$ & 0.25 \\
Heart rate $($ beats/min) & $133(126-147)$ & $135(127-147)$ & 0.59 \\
\hline Systolic BP $(\mathrm{mmHg})$ & $137(120-150)$ & $143(128-160)$ & 0.18 \\
\hline Hemoglobin $(\mathrm{g} / \mathrm{L})$ & $152(141-160)$ & $151(140-157)$ & 0.57 \\
\hline Creatinine $(\mu \mathrm{g} / \mathrm{L})$ & $83(69-94)$ & $77(68-88)$ & 0.32 \\
\hline CRP $(\mathrm{mg} / \mathrm{L})$ & $2.6(0.7-5.9)$ & $3.0(1.3-7.7)$ & 0.30 \\
\hline Glucose $(\mathrm{mmol} / \mathrm{L})$ & $6.4(5.9-7.7)$ & $7.0(6.1-9.2)$ & $\mathbf{0 . 0 4}$ \\
\hline Chest pain & $8(18 \%)$ & $11(24 \%)$ & 0.44 \\
\hline ST depression & $12(27 \%)$ & $10(22 \%)$ & 0.62 \\
\hline Cardioversion & $35(78 \%)$ & $32(71 \%)$ & 0.47 \\
\hline Echo data available & $39(87 \%)$ & $38(84 \%)$ & 0.76 \\
\hline
\end{tabular}

Data are presented as $\mathrm{n}(\%)$ of patients or median and 25th-75th interquartile range for continuous variables.

hsTnT: high-sensitivity troponin T; TIA: transient ischemic attack; Echo: echocardiography; CRP: c-reactive protein; AF: atrial fibrillation; AFL: atrial flutter; BP: blood pressure.

Higher age, hyperlipidemia and diabetes were associated with higher likelihood of hsTnT elevation at presentation (Table 1). We were not able to include diabetes in a multivariable logistic regression analysis because there were no patients with diabetes in the control group. Instead we used glucose levels at presentation, age and hyperlipidemia in the multivariable analysis, which showed that age and glucose levels were independently associated with hsTnT elevation at presentation (Table 2).

Table 2.

Significance of clinically relevant background factors in predicting elevated high-sensitivity troponin $\mathrm{T}$ at presentation

\begin{tabular}{lcc}
\hline & \multicolumn{2}{c}{ Multivariate analysis } \\
$\mathbf{n = 9 0}$ & OR (95\% Cl) & p-value \\
\hline Age (years) & $1.11(1.04-1.18)$ & 0.002 \\
Glucose (mmol/L) & $1.32(1.03-1.69)$ & 0.03 \\
\hline Hyperlipidemia & $2.7(0.83-8.7)$ & 0.10 \\
\hline
\end{tabular}

OR: odds ratio; Cl: confidence interval. 
The flow charts in Figure 1-2 and Table 3 demonstrates stress testing outcomes and the results of further evaluation. None of the controls reached the primary endpoint. Two of the cases reached the primary endpoint, of which one underwent elective $\mathrm{PCl}$ nearly three months after inclusion and the other had normal coronary arteries at subsequent coronary angiography. Two patients had inconclusive stress test results, but declined to be further evaluated.

Among the cases, hsTnT declined from a median of $25 \mathrm{ng} / \mathrm{L}$ (IQR 18 to $35 \mathrm{ng} / \mathrm{L}$ ) at inclusion in the ED to a median of $12 \mathrm{ng} / \mathrm{L}$ (IQR 8 to $15 \mathrm{ng} / \mathrm{L}$ ) at follow-up and $82 \%$ had either hsTnT below the 99th percentile or a significant $(>20 \%$ ) hsTnT decrease at follow-up (Table 3 ). During a follow-up period of 30 days, none of the patients, neither among the cases nor among the controls suffered a MACE (Table 3 ).

Table 3.

Troponin analyses, follow-up and outcomes.

\begin{tabular}{lccc}
\hline & $\begin{array}{c}\text { Controls } \\
(\mathbf{n = 4 5})\end{array}$ & $\begin{array}{c}\text { Elevated hsTnT } \\
(\mathbf{n = 4 5})\end{array}$ & $\begin{array}{c}\text { p- } \\
\text { value }\end{array}$ \\
\hline Baseline hsTnT (ng/L) & $6(4-8)$ & $12(8-15)$ & $<0.001$ \\
Peak hsTnT (ng/L) & $7(5-10)$ & $25(18-35)$ & $<0.001$ \\
\hline Significant $(>20 \%) \Delta$ hsTnT at follow-up or follow-up & & $37(82 \%)$ & \\
hsTnT $\leq 14$ ng/L & $15(33 \%)$ & $14(31 \%)$ & 0.82 \\
\hline Myocardial perfusion imaging & 0 & $2(4 \%)$ & 0.49 \\
\hline Primary endpoint & $9(20 \%)$ & $5(11 \%)$ & 0.25 \\
\hline Recurrence of arrhythmia & 0 & 0 & \\
\hline MACE during follow-up & & 0 \\
\hline
\end{tabular}

Data are presented as $n(\%)$ of patients or median and 25th-75th interquartile range for continuous variables.

hsTnT: high-sensitivity troponin T; MACE: major adverse cardiovascular events.

\section{Discussion}

As a result of the introduction of high-sensitivity cardiac troponins a lot of studies and reviews have been published focusing on the causes and consequences of minor hsTn elevations in various clinical contexts, emphasizing the need for further research [7, 21-24].

In this prospective study we aimed to address the quite common clinical problem of AF/AFL patients with RVR and dynamic hsTnT elevations.

The key finding of this study is that patients with paroxysmal AF/AFL presenting with a RVR and minor hsTnT elevations do not have an increased incidence of pathological stress tests compared to patients with hsTnT values below the 99th percentile. Of the two patients who reached the primary endpoint only 
one proved to have significant CAD. Since $82 \%$ of the patients either normalised or significantly decreased their troponin values within approximately one week, we conclude that most of the hsTnT elevations in this study is due to the acute tachyarrhythmia.

Costabel et al prospectively studied hsTnT elevations, in 100 patients with supraventricular tachycardia (84\% patients with AF or AFL), and its correlation to the presence of significant CAD. Approximately 50\% of the patients had elevated troponin (but only $15 \%$ of the patients with AF had significant hsTnT dynamics during serial testing) and four of these patients (all with AF) had a pathological stress test or were revascularized during 30 days of follow-up [25]. The reasons why they had slightly more patients with pathological stress tests or revascularisation in their study may be because they included more patients with chronically elevated hsTnT values and also that they included patients with known CAD.

Other previous studies addressing the significance of troponin elevations in AF patients are to our knowledge all retrospective, use the older $4^{\text {th }}$ generation troponin assays and come to different conclusions [2, 3, 26-28]. Some report an increased risk of $\mathrm{MI}$ and cardiac death during follow-up [2, 3, 27] while others fail to show that elevated troponin predicts significant CAD on angiography or adverse cardiac events during follow-up [26, 28]. Some of the reasons for this diversity may be different study populations and the use of different troponin assays with different cut offs (i.e. different absolute values define elevated troponin). Furthermore, there is probably a difference between acute and chronic hsTnT elevations and it is likely that this important difference cannot be distinguished in the different study populations.

Chronically elevated troponin values are, in a continuous way, a well-known predictor of cardiovascular morbidity and mortality [29-33]. At follow-up all controls and 32 out of 45 cases (71\%) had hsTnT below the 99th percentile indicating that our study population constitutes of AF/AFL patients with relatively low risk of adverse events. On the other hand, acute troponin elevations in a non-ACS setting are less studied and probably have different explanations in different contexts [7, 23]. For example, transient troponin elevations of unknown significance are reported in "harmless" conditions such as strenuous exercise [34] or in rapid atrial pacing [35]. But transient troponin elevations are also seen in sepsis, stroke and pulmonary embolism were troponin elevations have been shown to predict an adverse prognosis [7].

As previously mentioned, there are large knowledge gaps in this field of research. In this study we try to look at a specific clinical issue in a pragmatic way. We have also tried to address the same question in a previous retrospective study, where we could not find any strong correlation between dynamic hsTnT elevations in AF/AFL patients and significant CAD during follow-up [36], which is in line with the finding in this study. However, dynamic hsTnT elevations were associated with increased all-cause mortality, signalling presence of some kind of unknown pathophysiology [36]. Even if we acknowledge the fact that both the present and our previous study have methodological shortcomings, are relatively small and are underpowered to detect minor significant differences. Our results suggest that many of the observed hsTnT elevations, in patients without known HF and CAD presenting with AF/AFL and RVR, are not due to significant CAD caused supply-demand mismatch. 


\section{Limitations}

Firstly, two patients in the cases group had inconclusive stress tests and the results from possible further evaluation could have affected our outcomes.

Secondly, to be able to address our clinical problem and hypothesis as strictly as possible we used several inclusion and exclusion criteria. Consequently, our results should not be generalized to the entire population presenting with AF/AFL and RVR. However, we argue that AF/AFL patients without CAD, HF, renal failure and no other likely explanation for acute troponin elevation than AF/AFL with RVR are a real and clinically important problem that we address in this study.

Thirdly, we used a pathological bicycle exercise stress test as a surrogate marker of significant CAD. The reported sensitivity of exercise stress tests in relation to presence of significant CAD varies, but is less than $70 \%$ and one might argue that this is not good enough. However, in this population with a lowintermediate pre-test probability of CAD, no prior revascularization, predominantly normal resting ECGs and requirement to reach $\geq 85 \%$ of age-predicted maximum heart rate, the exercise stress test is recommended in current guidelines $[37,38]$ and a normal stress test is associated with an excellent prognosis [38]. Further, some patients primarily and all patients with inconclusive bicycle exercise tests (together approximately $30 \%$ of the study population) underwent evaluation with SPECT myocardial perfusion imaging, which make our results more reliable.

Fourthly, as previously mentioned, our study is not powered to show minor significant differences in our primary analysis. This is important to keep in mind when interpreting the results.

\section{Conclusions}

In this study, patients without known HF and CAD, presenting with acute paroxysmal AF/AFL with RVR and minor hsTnT elevations do not have an increased incidence of pathological stress test compared to patients with hsTnT values below the 99th percentile.

\section{Abbreviations}

ACS: acute coronary syndrome, AF: atrial fibrillation, AFL: atrial flutter, CABG: coronary artery bypass surgery, CAD: coronary artery disease, Cl: confidence interval, ECG: electrocardiogram, ED: emergency department, HF: heart failure, HsTn: high-sensitivity troponin, HsTnT: high-sensitivity troponin T, IQR: interquartile range, LBBB: left bundle branch block, MACE: major adverse cardiac event, MI: myocardial infarction, OR: odds ratio, PCl: percutaneous coronary intervention, RVR: rapid ventricular response, SPECT: single-photon emission computed tomography, TIA: transient ischemic attack, VEB: ventricular ectopic beat

\section{Declarations}


The study was conducted according to the principles of the Declaration of Helsinki and approved by the Regional Ethics Committee in Lund, Lund University (registry number 2014/453). All patients gave their informed consent.

Consent for publication

Not applicable

Availability of data and materials

The datasets used and analysed during the current study are available from the corresponding author on reasonable request.

Competing interests

The authors declare that they have no competing interests.

\section{Funding}

This work was supported by the Swedish National Research Council; the Swedish Heart-Lung Foundation; Novo Nordisk Foundation; Swedish Diabetes Association; Region Skåne, ALF; European Research Council grant [StG-2011-282255]; the Knut and Alice Wallenberg Foundation Clinical Scholars and Göran Gustafsson Foundation. But the funders had no part in the study design, data collection, data analysis, interpretation of data or in writing the manuscript.

\section{Authors' contributions}

JT and OM conceived the study and designed the trial. JT carried out the collection, analysis and interpretation of data and drafted the manuscript. OM and SG supervised the conduct of the trial and data collection. OM and SG participated in the analysis and interpretation of data and the drafting of the manuscript. All authors have read and approved the manuscript.

\section{Acknowledgements}

Not applicable.

\section{References}

1. Ball J, Carrington MJ, McMurray JJ, Stewart S: Atrial fibrillation: profile and burden of an evolving epidemic in the 21st century. Int J Cardiol 2013, 167(5):1807-1824.

2. Gupta K, Pillarisetti J, Biria M, Pescetto M, Abu-Salah TM, Annapureddy C, Ryschon K, Dawn B, Lakkireddy D: Clinical utility and prognostic significance of measuring troponin I levels in patients 
presenting to the emergency room with atrial fibrillation. Clin Cardiol2014, 37(6):343-349.

3. van den Bos EJ, Constantinescu AA, van Domburg RT, Akin S, Jordaens LJ, Kofflard MJ: Minor elevations in troponin I are associated with mortality and adverse cardiac events in patients with atrial fibrillation. Eur Heart $J$ 2011, 32(5):611-617.

4. Reichlin T, Hochholzer W, Bassetti S, Steuer S, Stelzig C, Hartwiger S, Biedert S, Schaub N, Buerge C, Potocki $\mathrm{M}$ et al: Early diagnosis of myocardial infarction with sensitive cardiac troponin assays. $N$ Engl J Med 2009, 361(9):858-867.

5. Keller T, Zeller T, Peetz D, Tzikas S, Roth A, Czyz E, Bickel C, Baldus S, Warnholtz A, Frohlich M et al: Sensitive troponin I assay in early diagnosis of acute myocardial infarction. N Engl J Med 2009, 361(9):868-877.

6. Hoff J, Wehner W, Nambi V: Troponin in Cardiovascular Disease Prevention: Updates and Future Direction. Curr Atheroscler Rep 2016, 18(3):12.

7. Agewall S, Giannitsis E, Jernberg T, Katus H: Troponin elevation in coronary vs. non-coronary disease. Eur Heart J 2011, 32(4):404-411.

8. Weijs B, Pisters R, Haest RJ, Kragten JA, Joosen IA, Versteylen M, Timmermans CC, Pison L, Blaauw $Y$, Hofstra $L$ et al: Patients originally diagnosed with idiopathic atrial fibrillation more often suffer from insidious coronary artery disease compared to healthy sinus rhythm controls. Heart Rhythm 2012, 9(12):1923-1929.

9. Nucifora G, Schuijf JD, van Werkhoven JM, Trines SA, Kajander S, Tops LF, Turta O, Jukema JW, Schreur JH, Heijenbrok MW et al: Relationship between obstructive coronary artery disease and abnormal stress testing in patients with paroxysmal or persistent atrial fibrillation. Int $J$ Cardiovasc Imaging 2011, 27(6):777-785.

10. Soliman EZ, Safford MM, Muntner P, Khodneva Y, Dawood FZ, Zakai NA, Thacker EL, Judd S, Howard VJ, Howard G et al: Atrial fibrillation and the risk of myocardial infarction. JAMA Intern Med 2014, 174(1):107-114.

11. Hansen CL, Goldstein RA, Akinboboye OO, Berman DS, Botvinick EH, Churchwell KB, Cooke CD, Corbett JR, Cullom SJ, Dahlberg ST et al: Myocardial perfusion and function: single photon emission computed tomography. J Nucl Cardiol 2007, 14(6):e39-60.

12. Thygesen K, Mair J, Giannitsis E, Mueller C, Lindahl B, Blankenberg S, Huber K, Plebani M, Biasucci LM, Tubaro $M$ et al: How to use high-sensitivity cardiac troponins in acute cardiac care. Eur Heart $J$ 2012, 33(18):2252-2257.

13. Fletcher GF, Ades PA, Kligfield P, Arena R, Balady GJ, Bittner VA, Coke LA, Fleg JL, Forman DE, Gerber TC et al: Exercise standards for testing and training: a scientific statement from the American Heart Association. Circulation 2013, 128(8):873-934.

14. Thygesen K, Alpert JS, Jaffe AS, Chaitman BR, Bax JJ, Morrow DA, White HD, Group ESCSD: Fourth universal definition of myocardial infarction (2018). Eur Heart J 2018.

15. Nordenfelt I, Adolfsson L, Nilsson JE, Olsson S: Reference values for exercise tests with continuous increase in load. Clin Physiol 1985, 5(2):161-172. 
16. Brudin $L$, Jorfeldt $L$, Pahlm O: Comparison of two commonly used reference materials for exercise bicycle tests with a Swedish clinical database of patients with normal outcome. Clin Physiol Funct Imaging 2014, 34(4):297-307.

17. Borg G, Ljunggren G, Ceci R: The increase of perceived exertion, aches and pain in the legs, heart rate and blood lactate during exercise on a bicycle ergometer. Eur J Appl Physiol Occup Physiol 1985, 54(4):343-349.

18. Banerjee A, Newman DR, Van den Bruel A, Heneghan C: Diagnostic accuracy of exercise stress testing for coronary artery disease: a systematic review and meta-analysis of prospective studies. Int J Clin Pract 2012, 66(5):477-492.

19. Enbergs A, Burger R, Reinecke H, Borggrefe M, Breithardt G, Kerber S: Prevalence of coronary artery disease in a general population without suspicion of coronary artery disease: angiographic analysis of subjects aged 40 to 70 years referred for catheter ablation therapy. Eur Heart J 2000, 21(1):45-52.

20. Brzezinski RY, Fisher E, Ehrenwald M, Shefer G, Stern N, Shapira I, Zeltser D, Berliner S, ShenharTsarfaty S, Milwidsky A et al: Elevated high-sensitive troponin $\mathrm{T}$ in negative stress test individuals. Eur J Clin Invest 2018, 48(6):e12930.

21. Alpert JS, Thygesen KA, White HD, Jaffe AS: Diagnostic and therapeutic implications of type 2 myocardial infarction: review and commentary. Am J Med 2014, 127(2):105-108.

22. Chapman AR, Adamson PD, Mills NL: Assessment and classification of patients with myocardial injury and infarction in clinical practice. Heart 2017, 103(1):10-18.

23. de Lemos JA: Increasingly sensitive assays for cardiac troponins: a review. JAMA 2013, 309(21):2262-2269.

24. Holzmann MJ: Clinical implications of high-sensitivity cardiac troponins. J Intern Med 2018, 284(1):50-60.

25. Costabel JP, Urdapilleta M, Lambardi F, Campos R, Vergara JM, Ariznavarreta P, Trivi M: HighSensitivity Cardiac Troponin Levels in Supraventricular Tachyarrhythmias. Pacing Clin Electrophysiol 2016, 39(6):588-591.

26. Parwani AS, Boldt LH, Huemer M, Wutzler A, Blaschke D, Rolf S, Mockel M, Haverkamp W: Atrial fibrillation-induced cardiac troponin I release. Int J Cardiol 2013, 168(3):2734-2737.

27. Conti A, Angeli E, Scorpiniti M, Alesi A, Trausi F, Lazzeretti D, Padeletti L, Gensini GF: Coronary atherosclerosis and adverse outcomes in patients with recent-onset atrial fibrillation and troponin rise. The American journal of emergency medicine 2015, 33(10):1407-1413.

28. Alghamry A, Hanna J, Pelecanos A, Kyranis S, Khelgi V, O'Rourke P, Carroll O, Oxenford C, Rangaswamaiah S, Tan C: Predictors of significant coronary artery disease in atrial fibrillation: Are cardiac troponins a useful measure. Int J Cardiol 2016, 223:744-749.

29. Saunders JT, Nambi V, de Lemos JA, Chambless LE, Virani SS, Boerwinkle E, Hoogeveen RC, Liu X, Astor BC, Mosley TH et al: Cardiac troponin T measured by a highly sensitive assay predicts coronary heart disease, heart failure, and mortality in the Atherosclerosis Risk in Communities Study. Circulation 2011, 123(13):1367-1376. 
30. de Lemos JA, Drazner MH, Omland T, Ayers CR, Khera A, Rohatgi A, Hashim I, Berry JD, Das SR, Morrow DA et al: Association of troponin T detected with a highly sensitive assay and cardiac structure and mortality risk in the general population. JAMA 2010, 304(22):2503-2512.

31. deFilippi CR, de Lemos JA, Christenson RH, Gottdiener JS, Kop WJ, Zhan M, Seliger SL: Association of serial measures of cardiac troponin T using a sensitive assay with incident heart failure and cardiovascular mortality in older adults. JAMA 2010, 304(22):2494-2502.

32. Hijazi Z, Oldgren J, Andersson U, Connolly SJ, Ezekowitz MD, Hohnloser SH, Reilly PA, Vinereanu D, Siegbahn A, Yusuf $S$ et al: Cardiac biomarkers are associated with an increased risk of stroke and death in patients with atrial fibrillation: a Randomized Evaluation of Long-term Anticoagulation Therapy (RE-LY) substudy. Circulation 2012, 125(13):1605-1616.

33. Hijazi Z, Wallentin L, Siegbahn A, Andersson U, Alexander JH, Atar D, Gersh BJ, Hanna M, Harjola VP, Horowitz JD et al: High-sensitivity troponin $\mathrm{T}$ and risk stratification in patients with atrial fibrillation during treatment with apixaban or warfarin. J Am Coll Cardio/ 2014, 63(1):52-61.

34. Gresslien T, Agewall S: Troponin and exercise. Int J Cardiol 2016, 221:609-621.

35. Turer AT, Addo TA, Martin JL, Sabatine MS, Lewis GD, Gerszten RE, Keeley EC, Cigarroa JE, Lange RA, Hillis LD et al: Myocardial ischemia induced by rapid atrial pacing causes troponin $T$ release detectable by a highly sensitive assay: insights from a coronary sinus sampling study. J Am Coll Cardiol 2011, 57(24):2398-2405.

36. Thelin J, Melander O: Dynamic high-sensitivity troponin elevations in atrial fibrillation patients might not be associated with significant coronary artery disease. BMC Cardiovasc Disord 2017, 17(1):169.

37. Task Force M, Montalescot G, Sechtem U, Achenbach S, Andreotti F, Arden C, Budaj A, Bugiardini R, Crea F, Cuisset $T$ et al: 2013 ESC guidelines on the management of stable coronary artery disease: the Task Force on the management of stable coronary artery disease of the European Society of Cardiology. Eur Heart J 2013, 34(38):2949-3003.

38. Miller TD: Stress testing: the case for the standard treadmill test. Curr Opin Cardiol 2011, 26(5):363369.

\section{Figures}




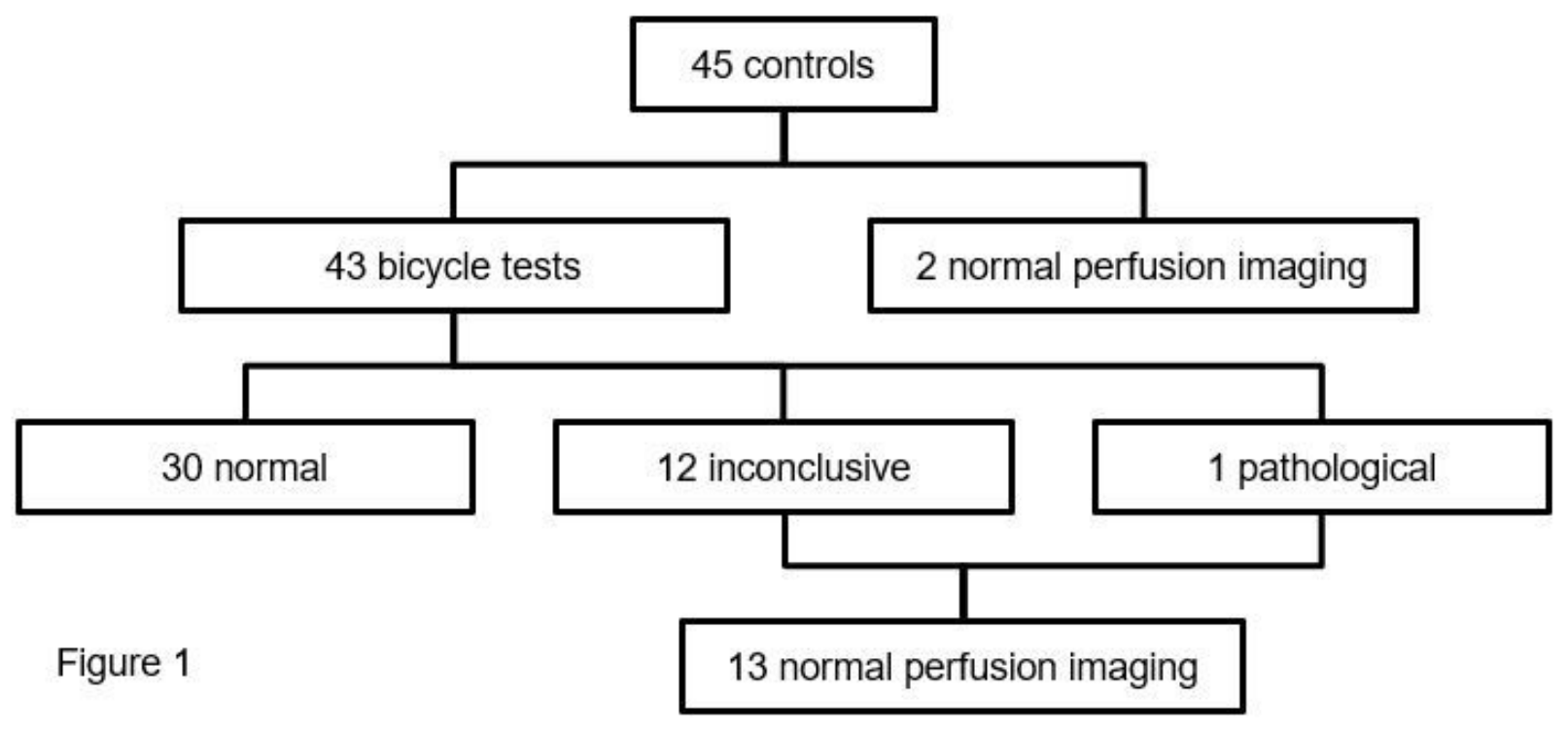

Figure 1

Outcomes and further evaluation in patients with hsTnT below the 99th percentile (controls)

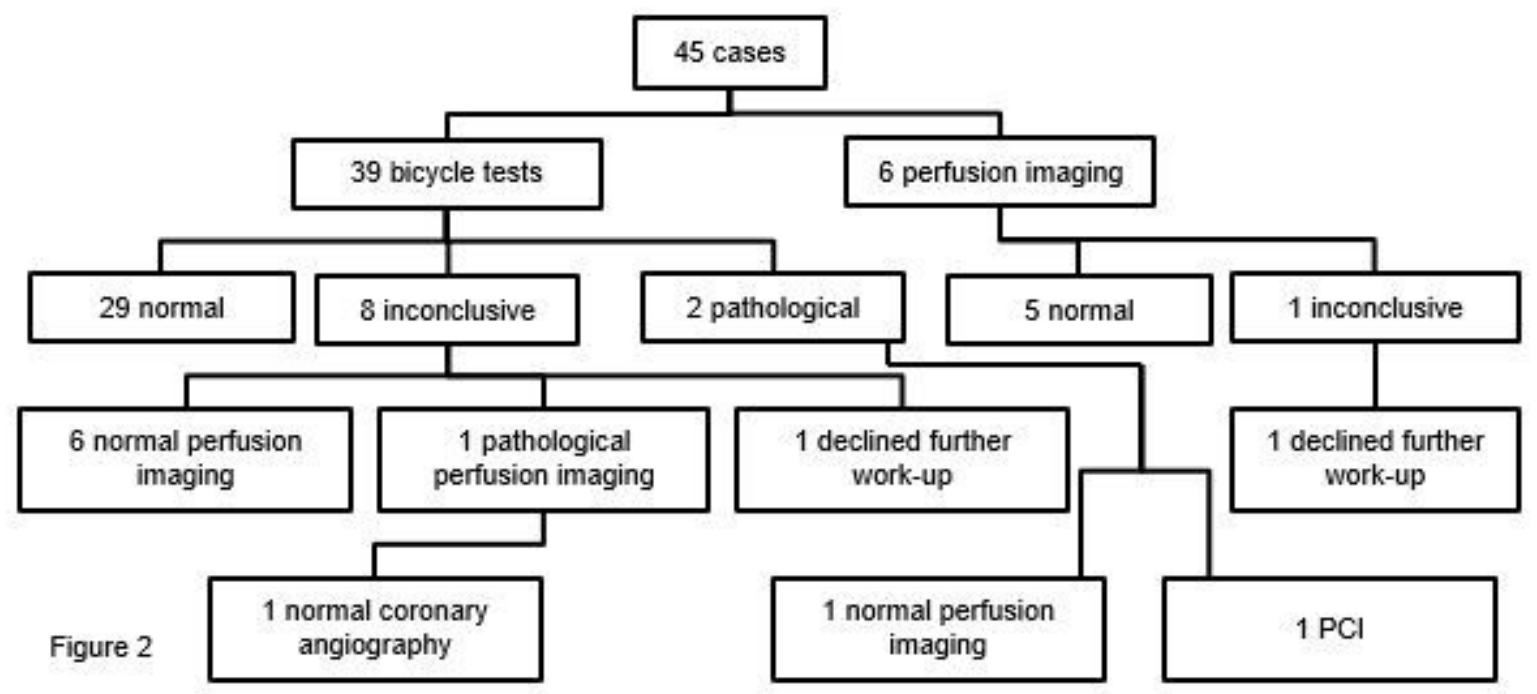

Figure 2

Outcomes and further evaluation in patients with elevated hsTnT (cases) PCl percutaneous coronary intervention 